\title{
DEFORMABLE TEMPLATE AND DISTRIBUTION MIXTURE-BASED DATA MODELING FOR THE ENDOCARDIAL CONTOUR TRACKING IN AN ECHOGRAPHIC SEQUENCE
}

\author{
Max Mignotte $\bullet^{\bullet}$ Jean Meunier $\bullet$ \\ - DIRO, Département d'Informatique et de Recherche Opérationnelle, P.O. 6128, Montréal (Québec), H3C 3J7. \\ $\ddagger$ INRIA, Institut National de Recherche en Informatique et Automatique, France. \\ e-mail: <mignotte,meunier>@iro.umontreal.ca
}

\begin{abstract}
We $e^{1}$ present a new method to shape-based segmentation of deformable anatomical structures in medical images and validate this approach by detecting and tracking the endocardial border in an echographic image sequence. To this end, a global prior knowledge of the endocardial contour is captured by a prototype template with a set of admissible deformations to take into account its inherent natural variability over time. In this approach, the data likelihood model rely on an accurate statistical modeling of the grey level distribution of each class present in the image. The parameters of this distribution mixture are given by a preliminary estimation step which takes into account the distribution shape of each class. Then the tracking problem is stated in a Bayesian framework where it ends up as an optimization problem. This one is then efficiently solved by a genetic algorithm combined with a steepest ascent procedure. This technique has been successfully applied on synthetic images and on a real echocardiographic image sequence.
\end{abstract}

\section{INTRODUCTION}

The segmentation of images is of great importance in medical imaging and remains a necessary step to obtain qualitative measurements such as the visualization or the location of objects of interest as well as for quantitative measurements such as area, volume or the analysis of dynamic behavior of anatomical structures over time. Among these images, ultrasound images play a crucial role, because they can be produced at video-rate and therefore allow a dynamic analysis of moving structures. Moreover, the acquisition of these images is non-invasive, cheap, and does not require radiations compared to other medical imaging techniques. On the other hand, the automatic segmentation of anatomical structures in ultrasound images is a real challenge due to speckle noise and artifacts which are inherent in these images.

Most current automatic segmentation techniques used in medical applications have been developed for CT and MRI images $[1,2]$. Among these segmentation schemes, two of the more reliable approaches use the Bayesian inference which allows to take into account an available local or global a priori knowledge. This available knowledge is then used to constraint the segmentation process. These methods are based on deformable templates or Markov Random Fields (MRF) models. Both have advantages and

\footnotetext{
${ }^{1}$ Acknowledgments: The authors thank INRIA (Institut National de la Recherche en Informatique et Automatique, France) for financial support of this work (postdoctoral grant).
}

shortcomings. The increased popularity of the deformable templates $[3,4]$ can be explained by their ability to express, via a global prior model, the geometric information and the shape variability of the object of interest to be detected over time and across individuals. In MRF-based segmentation approaches, the proper use of available prior information is expressed by a local prior model. This one expresses simply the fact that nearby pixels are fairly likely to belong to the same class [5].

The main problem with deformable models is that the likelihood energy are heuristically designed and do not statistically segment the image. As for MRF models, they cannot incorporate any global shape constraints in the segmentation process. Nevertheless, contrary to deformable model-based approaches, the likelihood model of an MRF model-based unsupervised segmentation exploits an accurate statistical modeling of the grey level distribution of each class present in the image [6]. Since it seems that deformable templates provide an interesting framework for structurally analyzing an image and MRF models provide a good mathematical setting for statistically analyzing an image, it makes sense to capitalize on the benefits of both methods.

To this end, we propose a deformable template based approach for the segmentation and the tracking of the endocardial contour in an echographic image sequence. We first define a prototype template along with a set of admissible transformations in order to capture the available global a priori shape knowledge of the endocardial contour with its inherent natural variability over time. In this Bayesian segmentation, the likelihood model rely on an accurate Markovian statistical modeling of the grey level distribution of each class present in the image. The parameters of this distribution mixture are given by a preliminary statistical estimation method called Iterative Conditional Estimation (ICE) [7]. Then, the detection and the tracking problem is stated in a Bayesian framework as the estimation of the deformation parameters of the template that maximize the posterior Probability Density Function (PDF). In order to efficiently maximize this function, we resort to a genetic exploration of the parameters space combined with a steepest ascent procedure.

This paper is organized as follows: Sections 2 and 3 present the template modeling and the proposed Bayesian approach. In Section 4 , we detail the distribution mixture parameters estimation step used in the data likelihood model. The stochastic search method using a hybrid Genetic Algorithm (GA) is described in Section 5. In Section 6, we report some experimental results on synthetic image and on a real ultrasound image sequence. Section 7 contains concluding remarks. 


\section{DEFORMABLE TEMPLATE REPRESENTATION}

In order to model the global shape knowledge of the endocardial contour, we first roughly define it by a set of $n$ labeled points, equally sampled, which approximate the outline of a circle (cf. Fig. 1.a). A cubic B-spline shape representation involving these $n$ control points corresponding to "landmarks" is then defined. The prototype template $\gamma_{0}$ does not describe the possible instances of the object shape to be detected. In order to take into account the natural variability of the considered object, we introduce a set of admissible affine and non-affine local and global transformations.

\section{Description of local non-affine transformations}

First, a local deformation process $\delta$ applied to the $n$ control points is introduced. These local deformations applied to the original shape $\gamma_{0}$ allow to model local random perturbations that can occur for each control point of the template outline. Mathematically, these local deformations are represented by local translations for each control point of $\gamma_{0}$ (cf. Fig. 1.b).

\section{Description of global non-affine transformations}

In order to refine the shape representation, we introduce a global non-affine deformation. The one used in this paper was first proposed by Jain et al. [8]. In this approach, the image is considered to be mapped to the unit square $S=[0,1]^{2}$. The deformation is then represented by a displacement function $\mathcal{D}(x, y)$. The mapping $(x, y) \mapsto(x, y)+\mathcal{D}(x, y)$ is thus a deformation of $S$, a smooth mapping of the unit square onto itself. The space of such displacement functions is spanned by the following orthogonal bases:

$$
\begin{aligned}
& e_{m n}^{x}(x, y)=(2 \sin (\pi n x) \cos (\pi m y), 0) \\
& e_{m n}^{y}(x, y)=(0,2 \cos (\pi n x) \sin (\pi m y))
\end{aligned}
$$

for $m, n=1,2, \ldots$ Low values of $m$ and/or $n$ correspond to lower frequency components of the deformation. The displacement function is then chosen as follows:

$$
\mathcal{D}_{\xi}(x, y)=\sum_{m=1}^{M} \sum_{n=1}^{N} \frac{\xi_{m n}^{x} e_{m n}^{x}(x, y)+\xi_{m n}^{y} e_{m n}^{y}(x, y)}{\lambda_{m n}}
$$

where $\lambda_{m n}=\pi^{2}\left(n^{2}+m^{2}\right)$ are the normalizing constants. The parameter vectors $\xi$ are the projections of the displacement function on the orthogonal basis previously defined. In order to allow a sufficiently wide range of possible deformations, while keeping the number of parameters reasonable, we use $M=N=2$. Fig. 1 illustrates the series of deformations of an initial template for different values of $M$ and $N$. We can notice that the deformation becomes more complex as higher frequency components are added to the displacement function $\mathcal{D}$.

\section{Description of affine transformations}

Finally, we introduce a set of admissible linear transformations on $\gamma$. These deformations involve translation, scaling, rotation, and stretching of the template.

Let now $\gamma_{\theta}$ be a deformed version of the original prototype $\gamma_{0}$ according to the aforementioned transformations with parameter vector $\theta$. A global configuration of the deformable template is thus described by 5 parameters corresponding to affine transformations, 4 pairs of global non affine deformation parameters, and $(n-2)$ pairs of translation vector $\left(\delta_{x_{i}}, \delta_{y_{i}}\right)$ respectively. The circular prototype template with the set of aforementioned transformations constitutes our global prior model describing the different possible configurations of the shape of interest.

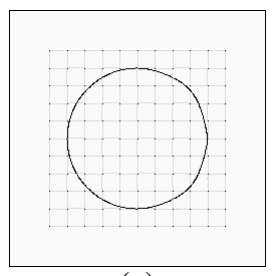

(a)

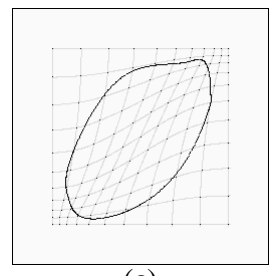

(c)

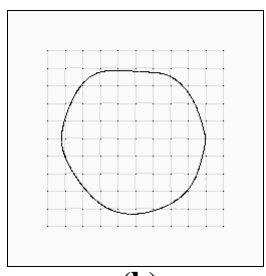

(b)

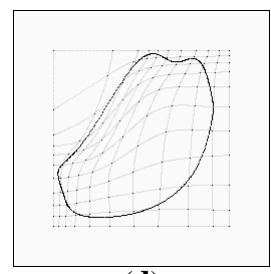

(d)
Figure 1: Local and global non-affine deformations. (a) Initial template (the grid is not part of the model itself). (b) $\gamma_{0}$ with local random perturbations. Global non-affine deformations on this locally deformed template with: (c) $M=N=1$ and $\xi_{m n}^{x}=\xi_{m n}^{y}=2 \forall m, n \in\{0,1\}^{2}$. (d) $M=N=2$ and $\xi_{m n}^{x}=\xi_{m n}^{y}=2 \forall m, n \in\{0,1,2\}^{2}$.

\section{SEGMENTATION MODEL}

\subsection{Introduction}

In a commonly used deformable model-based Bayesian segmentation approach, spatial and/or temporal gradient measure derived from the input image, are generally exploited in the data likelihood model $[1,8]$. Due to the speckle noise, our likelihood model cannot rely efficiently on this type of gradient measure. Instead, we propose a region oriented statistical approach. More precisely, we propose to model and use the grey level statistical distribution of each homogeneous region existing in an echogram and to exploit an a priori information about the location of the endocardium with respect to each other anatomical structures present in the ultrasound image. This a priori anatomical information expresses the fact that the endocardial contour delineates two homogeneous regions $\left\{\mathcal{R}_{0}, \mathcal{R}_{1}\right\}$. The first one, called the blood zone arises from the low acoustic wave reverberation in the ventricular cavity (filled with blood). The second one, called the muscle region $\left(\mathcal{R}_{1}\right)$, is due to the acoustic signal reverberation on the cardiac muscles. Each aforementioned region has completely different grey level intensity distribution and each pixel of the input image has a membership likelihood to each class $\left\{e_{0}=\right.$ blood, $e_{1}=$ muscle $\}$ that we can defined by the Probability Density Functions (PDFs) $P_{Y_{s} / X_{s}}\left(y_{s} / x_{s}=e_{i}\right) \quad i \in\{0,1\}$ where $y_{s}$ designates the grey level of the pixel at site $s$ and $x_{s}$ is its associated class label. Assuming that $N(N=2$ in our application) distinct homogeneous regions are present in the input image, the maximization over $\theta$ of the following expression:

$$
P_{Y / \Theta}(y / \theta)=\prod_{j=0}^{N-1} \prod_{s: x_{s}=e_{j}} P_{Y_{s} / X_{s}}\left(y_{s} / x_{s}\right)
$$

allows to find the optimal template (or the optimal parameter vector $\theta$ ) which best matches the image. Equivalently, we can minimize $-\ln P_{Y / \Theta}(y / \theta)$ and use this expression as our energy function. This energy function statistically segments the image in a Maximum Likelihood sense. 


\subsection{Joint model}

In that prospect, we let the template define two regions: the first one is the set of pixels inside the region delimited by the prototype template $\gamma_{\theta}$, and is denoted $\gamma_{\dot{\theta}}^{\bullet}$. The second one is the outside region, defined by the set of pixels located on the contour of the deformed template with a slightly superior scale. This last one is denoted $\gamma_{\theta}^{\circ}$. From this, we propose the following joint model through the Gibbs distribution:

$$
P_{\Theta, Y}(\theta, y)=\frac{1}{Z} \exp \{-\epsilon(\theta, y)\}
$$

$Z$ is a normalizing constant and $\epsilon$ is an objective function measuring how well a given instance of deformed template $\gamma_{\theta}$ fits the content of the input image $y$. The energy function $\epsilon(\theta, y)$ is composed of two terms as explained below:

- Likelihood energy term: this first energy term measures the likelihood of the image and is close to the log-likelihood distribution defined in equation (1).

$$
\begin{aligned}
\epsilon_{l}(\theta, y)= & -\frac{1}{N_{\gamma_{\theta}^{\bullet}}} \sum_{s \in \gamma_{\theta}^{\bullet}} \ln P_{Y_{s} / X_{s}}\left(y_{s} / e_{0}\right) \\
& -\frac{1}{N_{\gamma_{\theta}^{\circ}}} \sum_{s \in \gamma_{\theta}^{\circ}} \ln P_{Y_{s} / X_{s}}\left(y_{s} / e_{1}\right)
\end{aligned}
$$

where the summation of the first and second term of $\epsilon_{l}$ is over all the $N_{\gamma_{\theta}^{\bullet}}$ pixels of $\gamma_{\theta}^{\bullet}$ and over all the $N_{\gamma_{\theta}^{\circ}}$ pixels of $\gamma_{\theta}^{\circ}$ respectively. Note that the two weighting factors are necessary in order to get a scale-invariant likelihood measure.

- Prior energy term: the second term penalizes the deviation of the deformed template $\gamma_{\theta}$ from the original prototype $\gamma_{0}$ (see Section 2). This function does not penalize affine transformations and is close to the one proposed by Jain et al. in [8].

$$
\epsilon_{p}(\theta, y)=\left(\sum_{\xi_{i} \in \xi}\left[\left(\xi_{i}^{x}\right)^{2}+\left(\xi_{i}^{y}\right)^{2}\right]+\sum_{\delta_{i} \in \delta}\left(\delta_{i}\right)^{2}\right)
$$

\subsection{MAP detection}

Using these two energy terms, the joint distribution can be written as:

$$
P_{\Theta, Y}(\theta, y)=\frac{1}{Z} \exp -\left\{\epsilon_{l}(\theta, y)+\rho \epsilon_{p}(\theta, y)\right\}
$$

where the factor $\rho$ provides a relative weighting between the two penalty terms and allows to control the "rigidity" of the template. The posterior distribution deduced from (2) is given by:

$$
P_{\Theta / Y}(\theta / y)=\frac{1}{Z_{y}} \exp \{-\epsilon(\theta, y)\}
$$

where $Z_{y}$ is a normalizing constant depending on $y$ only. We formulate now the detection problem as the search of the Maximum $A$ Posteriori (MAP) estimation of $\theta$ :

$$
\begin{aligned}
\hat{\theta}_{M A P} & \in \arg \max _{\theta}\left\{P_{\Theta / Y}(\theta / y)\right\} \\
& \in \arg \min _{\theta} \epsilon(\theta, y)
\end{aligned}
$$

The search of $\hat{\theta}_{M A P}$ is equivalent to the minimization of $\epsilon$. This function is minimal when the deformed template delineates two homogeneous regions distributed according to the grey level distribution corresponding to each region (via $\epsilon_{l}$ ) and when the deformed template is not too different from the original prototype (via $\epsilon_{p}$ ). In order to get an unsupervised deformable-based segmentation scheme, we have now to estimate the distribution mixture parameters. We consider this in the next section.

\section{MIXTURE PARAMETER ESTIMATION}

\subsection{ICE procedure}

Consider now a couple of random fields $Z=(X, Y)$, where $Y=$ $\left\{Y_{s}, s \in S\right\}$ represents the field of observations located on a lattice $S$ of $N$ sites $s$ (associated to the $N$ pixels of the image), and $X=\left\{X_{s}, s \in S\right\}$ the label field (related to $N$ blood or muscle class labels of the segmented image). Each $Y_{s}$ takes its value in $\{0, \ldots, 255\}$, and each $X_{s}$ in $\left\{e_{0}=\right.$ blood, $e_{1}=$ muscle $\}$. The distribution of $(X, Y)$ is defined, firstly, by prior distribution $P_{X}(x)$, supposed to be stationary and Markovian in this mixture parameter estimation step, and secondly, by site-wise likelihoods $P_{Y_{s} / X_{s}}\left(y_{s} / x_{s}\right)$, depending on class label $x_{s}$. The observable $Y$ is called the "incomplete data", and $Z$ the "complete data". The conditional likelihood $P_{Y / X}(y / x)$ depends on parameter vector $\Phi_{y}$, which is the distribution mixture parameter vector to be estimated. In order to obtain a reliable estimation of this parameter, we resort to the ICE algorithm [9]. This method relies on an estimator $\hat{\Phi}_{y}(x, y)$ for completely observed data case. When $X$ is unobservable, this procedure starts from an initial parameter vector $\Phi_{y}^{[0]}$ and generates a sequence of parameter vectors leading to the optimal parameters. To this end, $\Phi_{y}^{[k+1]}$ at step $(k+1)$ is chosen as conditional expectation of $\hat{\Phi}_{y}$ given $Y=y$, computed according to the current value $\Phi_{y}^{[k]}$. It is the best approximation of $\Phi_{y}$ in terms of the mean squares error [9]. By denoting $E_{k}$, the expectation relative to parameter vector $\Phi_{y}^{[k]}, \Phi_{y}^{[k+1]}$ is computed from $\Phi_{y}^{[k]}$ and $Y=y$ by:

$$
\Phi_{y}^{[k+1]}=E_{k}\left[\hat{\Phi}_{y}(X, Y) \mid Y=y\right]
$$

The computation of this expectation is impossible in practice, but we can approach it thanks to the law of large numbers by:

$$
\Phi_{y}^{[k+1]}=\frac{1}{n}\left[\hat{\Phi}_{y}\left(x_{(1)}, y\right)+\cdots+\hat{\Phi}_{y}\left(x_{(n)}, y\right)\right]
$$

where $x_{(i)}, i=1, \ldots, n$ are realizations of $X$ drawn according to the posterior distribution $P_{X / Y, \Phi_{y}}\left(x / y, \Phi_{y}^{[k]}\right)$. As explained below, for complete data estimator $\hat{\Phi}_{y}(x, y)$, we use a Maximum Likelihood (ML) estimator for the noise model parameter. In order to decrease the computational load, we can take $n=1$ without altering the quality of the estimation [7]. Finally, we can use the Gibbs sampler algorithm [10] to simulate realizations of $X$ according to the posterior distribution.

\subsection{Estimation of $\Phi_{y}$ for the complete data}

In order to take into account the speckle noise phenomenon [11] in the reverberation areas, we model the conditional PDF of each class by a shifted Rayleigh law:

$$
\mathcal{R}_{Y}(y ; \min , \alpha)=\frac{y_{s}-\min }{\alpha^{2}} \exp \left(-\frac{\left(y_{s}-m i n\right)^{2}}{2 \alpha^{2}}\right)
$$

with $y>\min$ and $\alpha>0$. Let now $Y=\left(Y_{1}, \ldots, Y_{M}\right)$ be $M$ random variables, independent and identically distributed according 
to a single Rayleigh law $\mathcal{R}_{Y}\left(y ; \Phi_{y}\right)$, and $y=\left(y_{1}, \ldots, y_{M}\right)$ a realization of $Y$. The ML estimators of $\Phi_{y}$ for the "complete data" of this sample are given by [12]:

$$
\begin{aligned}
\widehat{\min }_{M L} & \approx \hat{y}_{\min }-1 \\
\hat{\alpha}_{M L}^{2} & =\frac{1}{2 M} \sum_{i=1}^{M}\left(y_{i}-\widehat{\min }_{M L}\right)^{2}
\end{aligned}
$$

where $\hat{y}_{\min }=\min _{i}\left(y_{i}\right)$.

\subsection{Estimation of $\Phi_{y}$ for the incomplete data}

For the local a priori model of the Gibbs sampler, we adopt a standard isotropic Potts model with the 8-connexity spatial neighborhood [5]. In this model, there are four parameters, called "the clique parameters" associated to the horizontal, vertical, right and left diagonal binary cliques respectively. Given this local a priori model, the prior distribution $P_{X}(x)$ can be written as:

$$
P_{X}(x)=\exp \left\{-\sum_{<s, t>} \beta_{s t}\left(1-\delta\left(x_{s}, x_{t}\right)\right)\right\}
$$

where summation is taken over all pairs of neighboring sites and $\delta($.$) is the Kronecker delta function. In order to favor homoge-$ neous regions with no privileged orientation in the Gibbs sampler simulation process, we choose $\beta_{s t}=1$. The parameter estimation procedure for the incomplete data is outlined below:

Parameter initialization: The initial parameter values have a significant impact on the convergence of the ICE procedure and on the quality of the final estimates. We can use the initialization method described in [6] or give a parameter vector not "too far" from the optimal one.

ICE procedure: $\Phi_{y}^{[k+1]}$ is computed from $\Phi_{y}^{[k]}$ in the following way:

1. Using the Gibbs sampler, one realization $x$ is simulated according to the posterior distribution based on $\Phi_{y}^{[k]}$.

2. $\Phi_{y}^{[k+1]}$ is estimated with the ML estimator $\hat{\Phi}_{y}(x, y)$ of the "complete data".

3. If the sequence $\Phi_{y}$ becomes steady, the ICE procedure is ended, else we return to step 1 .

Fig. 2 represents the distribution mixture estimated on the echogram shown in Fig.3.a and the histogram of this ultrasound image: the two site-wise likelihoods are superimposed to the image histogram. Corresponding estimates obtained by the ICE procedure are given in Table 1 . The quality of the estimations is difficult to appreciate in absence of ground truth values. We can roughly perform such an evaluation by comparing the image histogram with the PDF corresponding parameters. Estimation results on synthetic images with ground truth values will be given in Section 6 in order to appreciate the robustness of this procedure and the quality of the estimations. Experiments have shown that the distribution mixture parameters keep constant for all the images extracted of the same echographic sequence. For this reason

\begin{tabular}{|c|c|c|c|}
\hline & \multicolumn{3}{|c|}{$\begin{array}{l}\text { ECI Procedure } \\
\end{array}$} \\
\hline$\Phi_{y_{(\text {blood })}}^{\text {final }}$ & $0.48_{(\pi)}$ & $24_{(\min )}$ & $207_{\left(\alpha^{2}\right)}$ \\
\hline$\Phi_{y_{(\text {muscle })}}^{\text {ninal }}$ & $0.52_{(\pi)}$ & $24_{(\min )}$ & $9436_{\left(\alpha^{2}\right)}$ \\
\hline
\end{tabular}
the mixture parameters are computed once and for all on the first frame of the echographic sequence.
Table 1: Estimated parameters for the picture reported in Fig.3.a. $\pi$ stands for the proportion of the two classes within the image. min and $\alpha$ are the shifted Rayleigh law parameters.

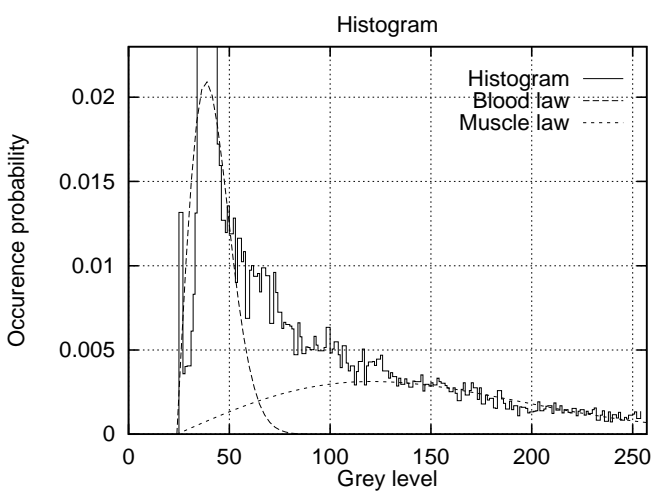

Figure 2: Image histogram of the picture reported in Fig.3.a (solid curve) and estimated PDF mixture obtained with the ICE procedure (dashed and dotted curves).

\section{OPTIMIZATION PROBLEM}

The objective function $\epsilon(\theta, y)$ to be minimized is complex with several local extrema over the deformation parameter space. A global search is usually impossible due to the size of the configuration space. Instead, we have implemented a Genetic Algorithmbased optimization technique [13] with the following fitness measure $\mathcal{F}$ (to be maximized):

$$
\mathcal{F}(\theta)=\exp \{-\epsilon(\theta, y)\}
$$

In order to speed up the convergence rate, we have developed two strategies and we have combined them. The first one is an elitepreservation strategy [13]: the individual with the highest fitness survives to be an individual of the next generation. The second strategy (called hybrid GA [13]) consists in associating the genetic search with a local optimization technique. In each generation, a percentage of the best individuals are used to initialize a gradient ascent technique. Therefore, these best individuals explore local neighborhoods in the parameter space to find a point of higher fitness.

\section{EXPERIMENTAL RESULTS}

Distribution mixture estimation step: based on the distribution mixture parameters given by the ICE procedure (see Table 1), we can compute the probability map for the blood and muscle classes. This map exhibits sharp boundaries between high and low probability regions for each class. As a result, the probability maps have a high gradient at the boundary of the different regions and low gradient everywhere else. In order to constraint the deformable model to be efficiently attracted to the boundaries of two homogeneous regions by the gradient-based local optimization procedure, we need to spread their region of influence to a larger area. To do this, we convolve each probability map by a 2D Gaussian mask. 
Detection step: in order to reduce the size of the parameter search space, the GA-based stochastic exploration is used to only estimate the affine transformation parameters (five parameters to be estimated). This affine deformation parameter estimation ensures a first crude registration of the shape to be detected. The local exploration technique of the best individuals selected by the $\mathrm{GA}$, which is based on the steepest ascent procedure, is then used to estimate the whole parameter vector.

Tests have shown that this optimization procedure is not very sensitive to the control parameters. In our application, these parameters are commonly used [13] and are the following: population size $=100$, crossover rate $=0.8$, mutation rate $=0.008$, maximum number of generations $=30$. At each generation, we select five best individuals (i.e., parameter vectors) for the hybridation with the local optimization technique. The prototype template is a connected 12-points model. The weighting factor penalizing $\epsilon_{p}$ term with respect to $\epsilon_{l}$ term is set to 0.1 . All these parameters keep constant for the detection and the tracking step.

Using this procedure, a reliable detection of the endocardial contour is obtained for all images of the echographic sequence. Our GA takes about 5-20 generations to converge to the true solution. In fact, the convergence rate can vary depending on the complexity of $\epsilon$. Tests have shown that, after a learning step (i.e., typically 3-6 generations of the GA), the genetic exploration provides good initializations for the local exploration technique. In our application, the optimization procedure for the detection process takes about three minutes (average CPU time) on a standard Sun/Sparc2 workstation. Fig. 3 shows the first frame of the echographic sequence and illustrates the best deformed template for successive generations of the genetic search. In spite of the speckle noise and a random initial population for the GA, an accurate and reliable detection of the endocardial border is obtained. Fig. 4 shows a synthetic image with synthetic speckle noise and the resulting detection/segmentation obtained by our method. Real parameters of the distribution mixture can be compared to estimated parameters by the ICE procedure (see Table 2). We can notice that faithful noise model estimations and accurate segmentation are obtained.

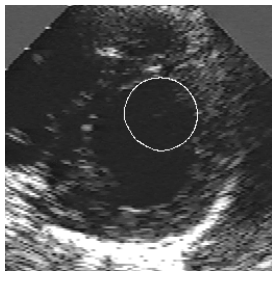

(a)

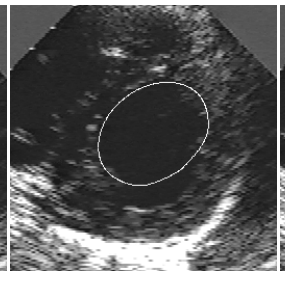

(b)

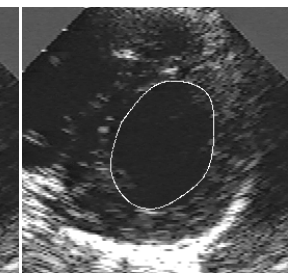

(c)
Figure 3: Best resulting template after: (a) $2(\mathcal{F}(\epsilon)=0.091)$. (b) $3(\mathcal{F}(\epsilon)=0.119)$. (d) 8 generations $(\mathcal{F}(\epsilon)=0.186)$.

Tracking step: the tracking strategy used in our application is the following: the final estimate of the previous time frame is used as an initialization for the steepest ascent procedure for the current time frame. If the resulting value of energy $\epsilon$ at convergence is higher than a given threshold, we can assume that the inter-frame motion is small and this strategy allows to provide a proper initialization of the template for the local exploration technique used on the next frame. In this case, the result of the gradient ascent technique gives the final result for the current time frame.

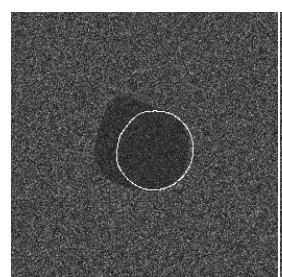

(a)

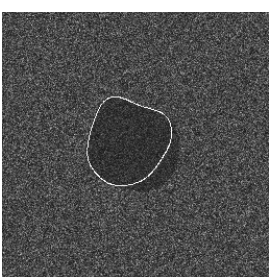

(b)

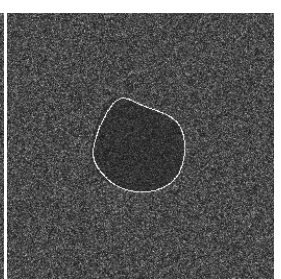

(c)
Figure 4: Best resulting template after: (a) $2(\mathcal{F}(\epsilon)=0.149)$. (b) $4(\mathcal{F}(\epsilon)=0.166)$. (d) 10 generations $(\mathcal{F}(\epsilon)=0.174)$.

\begin{tabular}{llll}
\hline \hline & \multicolumn{3}{c}{ ICE procedure } \\
\hline$\Phi_{y_{\left(e_{0}\right)}}^{\text {final }}$ & $0.09_{(\pi)}$ & $19_{(\min )}$ & $672_{\left(\alpha^{2}\right)}$ \\
\hline$\Phi_{y_{\left(e_{1}\right)}}^{\text {tinal }}$ & $0.91_{(\pi)}$ & $19_{(\min )}$ & $2037_{\left(\alpha^{2}\right)}$ \\
\hline \hline \multicolumn{4}{c}{ Real parameters } \\
\hline$\Phi_{y_{\left(e_{0}\right)}}^{\text {real }}$ & $0.09_{(\pi)}$ & $19_{(\min )}$ & $700_{\left(\alpha^{2}\right)}$ \\
\hline$\Phi_{y_{\left(e_{1}\right)}}^{\text {real }}$ & $0.91_{(\pi)}$ & $19_{(\min )}$ & $2000_{\left(\alpha^{2}\right)}$ \\
\hline \hline
\end{tabular}

Table 2: Estimated parameters by the ICE procedure and real parameters for the picture reported in Fig. 4. $\pi$ stands for the proportion of the two classes within the synthetic image. min and $\alpha$ are the Rayleigh law parameters.

Otherwise, we decide to use the genetic algorithm combined with the steepest ascent procedure to re-estimate the whole parameter vector $\theta$. To this end, each of the $L$ parameters $\theta_{l}$ is quantified on 8 bits in order to take any value within the range $\left[\theta_{l}-\delta \theta_{l}, \theta_{l}+\delta \theta_{l}\right]$ with $\theta_{l}$ the estimated parameter value given at the previous time frame.

Fig. 5 and 6 show the tracking of the endocardial contour in a medical echographic sequence at different time frames. We can notice that the proposed method is reliable and the used global prior model seems to be flexible enough for representing accurately the inherent natural variability of the endocardial contour during the cardiac cycle.

\section{CONCLUSION}

In this paper, we have developed a robust algorithm to detect and track anatomical deformable structures like the endocardial border in an ultrasound image sequence. We have stated this problem in the Bayesian framework and taken into account all the available a priori knowledge of this problem. First, the proposed global prior model has shown itself to be very flexible and well suited to model the high variability of the endocardial contour over long image sequence. Another important source of a priori knowledge is information about the intensity of the tissue of different anatomical structures. These factors, highly dependent on the imaging process, are taken into consideration by modeling the distribution of each class of the ultrasound image by a Rayleigh law. Parameters of each PDF are given by a preliminary estimation step and are exploited in the data likelihood model. This method seems to be particularly well suited to handle ultrasound images with strong speckle noise on which edge information cannot be exploited. Finally, the local and global minimization procedure we propose is fast, robust and do not require initialization of the template close to the desired solution. Initialization may be defined at random, leading to segmentation and tracking procedure that are completely data driven. 


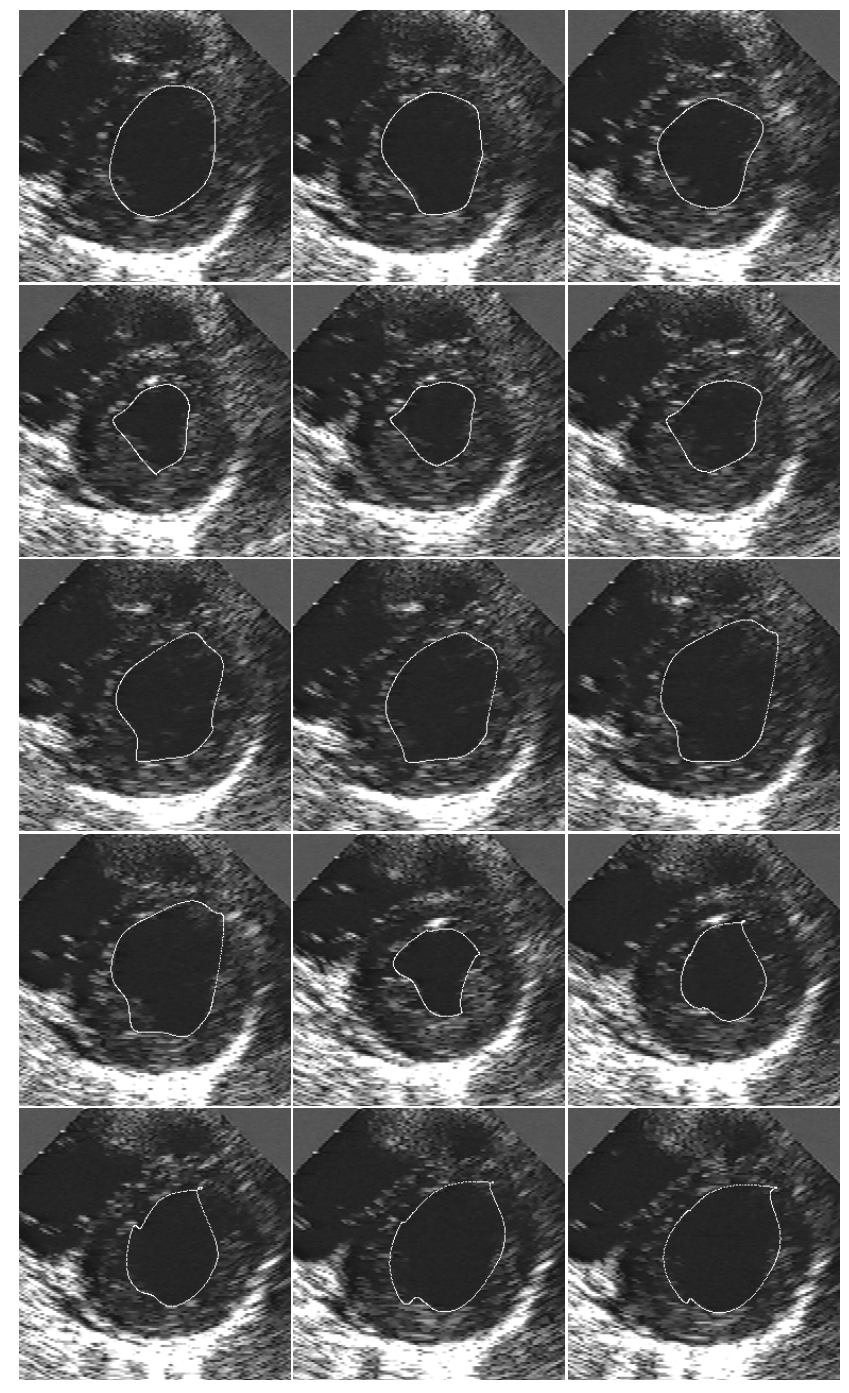

Figure 5: Tracking of the endocardial contour in a medical echographic sequence at different time frames during the cardiac cycle. From top left to bottom right : frame 1, 4, 6, 9, 12, 13, 18, 20, 27, 30, 35, 40, 41, 44, 46.

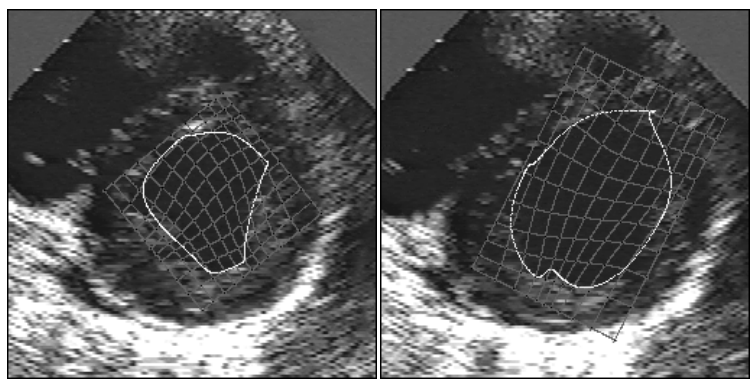

(a)

(b)

Figure 6: Detection/segmentation obtained with our procedure on: (a) frame 33. (b) frame 45.

\section{REFERENCES}

[1] C. Kervrann and F. Heitz. Statistical model-based segmentation of deformable motion. In Proc. ICIP, pages 937-940, Lausanne, 1996.

[2] S.D. Fenster and J.R. Kender. Sectored snakes: evaluating learned-energy segmentations. In Proc. ICCV, pages 420426, Jan. 1998.

[3] J. Feldmar and N. Ayache. Rigid, affine and locally affine registration of free form surfaces. The International Journal of Computer Vision, 18(2), 1996.

[4] J. Montagnat and H. Delingette. Globally constrained deformable models for $3 \mathrm{~d}$ object reconstruction. Signal Processing, 71(2):173-186, 1998.

[5] J. Besag. On the statistical analysis of dirty pictures. Journal of the Royal Statistical Society, B-48:259-302, 1986.

[6] M. Mignotte, C. Collet, P. Pérez, and P. Bouthemy. Unsupervised Markovian segmentation of sonar images. In Proc. ICASSP, volume 4, pages 2781-2785, Munchen, May 1997.

[7] B. Braathen, P. Masson, and W. Pieczynski. Global and local methods of unsupervised Bayesian segmentation of images. GRAPHICS and VISION, (1):39-52, 1993.

[8] A.K. Jain, Y. Zhong, and S. Lakshmanan. Object matching using deformable templates. IEEE Trans. on PAMI, 18(3):267-278, 1996.

[9] F. Salzenstein and W. Pieczinsky. Unsupervised Bayesian segmentation using hidden markovian fields. In In proc. ICASSP, pages 2411-2414, May 1995.

[10] S. Geman and D. Geman. Stochastic relaxation, Gibbs distributions and the Bayesian restoration of images. IEEE Trans. on PAMI, 6(6):721-741, 1984.

[11] J. W. Goodman. Some fundamental properties of speckle. Journal of Optical Society of America, 66(11):1145-1150, 1976.

[12] F. Schmitt, M. Mignotte, C. Collet, and P. Thourel. Estimation of noise parameters on sonar images. In Signal and Image Processing, volume SPIE 2823, pages 1-12, Denver, USA, Aug. 1996.

[13] D.E Goldberg. Genetic Algorithm. Addison Wesley, 1989. 